Grant \# DE-FG03-88ER45375

T.P.Russell

IBM Almaden Research Center

650 Harry Road

San Jose, CA 95120

\title{
Research Activities
}

\section{Grazing Incidence Diffraction on Polyalkylsilanes}

In an extension of the grazing incidence $x$-ray scattering work on aromatic polyimides, the effect of an interface on the structure of a more rigid molecules, polv(hexyl pentyl silane) was investigated. While the Si backbone is as flexible as $\mathrm{C}$ backbone, the substitution of hulky sidegroups onto the Si chain forces the molecule to adopt a rigid conformation. In fact, in the bulk, the PHPS, at room temperature, is liquid crystalline with the polymer chains packing in an hexagonal manner. GIXS studies on films of PHPS have clearly shown that PHPS molecules are oriented parallel to the air and substrate interfaces with the hexagonal packing of the molecules propagating throughout the entire thickness of the sample. Near the surface, there is a slight rotation of the hexagonal lattice by approximately $30^{\circ}$ which results from density constraints. In addition, the order along the chain axis is dramatically enhanced.

\section{Grazing Incidence Scattering on Flexible Chains}

Grazing incidence scattering studies were performed on a perfluoropolyether, a polymer chain that is, in the bulk, liquid at room temperature. Studies were performed on solution deposited films with a thickness of $100 \AA$. To within $50 \AA$ from the air surface, the density correlations parallel and perpendicular to the surface were found to be same and identical to those found in a bulk sample. Consequently, to within the depth resolution of these studies, no evidence was found for enhanced ordering near an interface. Studies were performed as a function of the film thickness also. Down to a film thickness of $13 \AA$, only a few molecular diameters, the density correlations parallel and perpendicular to the film surface and were also found to be identical to that found in the bulk. There is absolutely no question, that substantial ordering of the molecules did not occur, even for the thinnest films. This is the first demonstration that such ordering does not occur. However, these results do not precluded the formation of a nematic ordering of the chains since the density correlations would not undergo dramatic changes. In addition, the measurements are time averaged, and whether the dynamics of the molecules are altered by these severe restraints is still an open question.

\section{Chain Distributions in Lamellar Microdomains}

It has been shown previously that thin films of diblock copolymers of polystyrene and poly(methyl methacrylate), denoted $\mathrm{P}$ (S-b-MMA), form a multilayered morphology with the lamellar microdomains oriented parallel to the air and substrate interfaces. Using P(S-b-MMA) where either the junction points or the chain ends were labelled with deuterium, neutron reflectivity studies provided a quantitative measure of the configuration of the copolymer chains in the lamellar microdomains. From the scattering length density 


\section{DISCLAIMER}

Portions of this document may be illegible in electronic image products. Images are produced from the best available original document. 
profiles derived from the reflectivity measurements, the spatial distribution of the junction points and the chain ends could be directly determined. It was found that the junction points were almost totally confined to the interfacial region as would be expected. However, the chain ends were found to be distributed throughout the lamellar microdomain. While there was a concentration of chain ends in the center of the domain, there was clear evidence that the chain ends also penetrated into the interfacial region. In addition, the distribution of chain ends at the air and substrate interfaces were identical to that seen in the interior of the sample.

\section{Time Dependent Ordering of Copolymers}

The ordering of symmetric, diblock copolymers of PS and PMMA, denoted P(S-b-MMA) on Au substrates was studied by transtnission electron microscopy, TEM. Films of P(S-b-MMA) were cast onto Au coated polyimide substrates and annealed. Samples were then cross sectioned normal to the film surface and stained with $\mathrm{RuO}_{4}$. The as cast $\mathrm{P}(\mathrm{S}-\mathrm{b}-\mathrm{MMA})$ films were found to microphase separate into a highly disordered structure with a period $\sim 1 / 3$ that of the equilitrium value. Upon annealing, lamellar microdomains having the equilibrium period organized into lamellar microdomains oriented parallel to the free surface with a persistence which increased with further annealing. However, at the Au interface, effectively a neutral interface, two different orientations of the lamellar microdomains were found, i.e. either parallel to or normal to the interface. In very thin films, if the vertical orientation began at the substrate interface, this orientation was found to dominate the thin film morphology. Regardless of the orientation of the lamellae, PS was found to fully cover the air surface.

\section{A New Phase Transition In Diblock Copolymers}

The order disorder transition of symmetric, diblock copolymers of polystyrene and poly(n-butyl methacrylate), denoted $P($ S-b-nBMA), was investigated by small angle $x$-ray and neutron scattering. SAXS and SANS, respectively. For P(S-b-nBMA) with $\mathrm{MW}=9.9 \times 10^{4}$ a typical order-disorder transition was observed near $100^{\circ} \mathrm{C}$. However, upon increasing the temperature further, a disorder to order transition was found. The period of the lamellar microdomain morphology at the elevated temperatures was found to be nearly the same as that observed at the lower temperatures, taking into account thermal expansion. For $\mathrm{P}(\mathrm{S}-\mathrm{b}-\mathrm{nBMA})$ with $\mathrm{MW}=3.4 \times 10^{4}$, the order to disorder transition was suppressed below ambient temperature and the disorder-order transition was found to be elevated. Thus, with decreasing molecular weight the temperature range for the disordered state is expanded. These results provide evidence of a microphase separation of a diblock copolymer driven by the thermal expansion of the system. In analogy with binary polymer mixtures, equation of state arguments can be used to explain the LDOT, the lower disorder to order transition.

\section{Thermal Expansion of Thin Film Diblock Copolymers}

The thermal expansion of symmetric diblock copolymers of PS and PMMA, denoted $P(S-b-M M A)$ was investigated by $x$-ray reflectivity. The refiectivity profile contains interferences from which the total film thickness and the period of the lamellar microdomains (oriented parallel to the surface) can be determined. Upon heating the fim thickness and lamellar period increased by an amount commensurate with the rolume expansion coefficient. This, due to the confinement of the copolymer on the substrate, is a factor of three greater than that experienced by diblock copolymer in the bulk. Consequently, the chain is stretched significantly at the interface between the lamellar microdomains. With time, at elevated temperature, the period was found to decrease which produces a relaxation of the chains at the interface. This is done by forcing copolymer 
chains to the surface which represents a balance between the enthalpy of mixing dissimilar chains and elastic, retractive forces. 
Proposed Research Plans for 1993-1994

\section{Overview}

Over the past several years at least four different techniques have been developed for probing the behavior of homopolymers and block copolymers at surfaces and interfaces. These include neutron reflectivity, graxing incidence $x$-ray scattering, dynamic secondary ion mass spectrometry and electron microscopy. In the upcoming year it is proposed to make key uses of these developments to gain a quantitative understanding of the interfacial behavior of homopolymers and block copolymers. In addition, the newly discovered lower disorder to order phase transition will be investigated further. is the role a surface or an interface plays in influencing the structure and segmental distribution of polymers in the vicinity of an interface. These areas are outlined below.

\section{Copolymers at Homopolymer Interfaces}

By a combination of neutron reflectivity and dynamic secondary ion mass spectroscopy, the segment density profiles of copolymers at the homopolymer interfaces will be determined. It has been shown that a quantitative description of the segment density profiles can be determined using these techniques combined with a selective labelling of the copolymers and homopolymers with deuterium. It is proposed to extend these studies by determining the effect of the homopolymer molecular weight on the extent to which the copolymer molecules penetrate into the homopolymer layers. These studies have been initiated but, by lack of sufficient neutron beam time, the studies have not been completed. It is proposed to investigate the case where the molecular weight of the homopolymer is varied by $\sim 100$. In addition, these studies will be extended further to the case where the homopolymers are different than the blocks of the copolymer. Herein, the enthalpic swelling of the copolymer chains at the interface can be systematically probed by varying the segmental interaction parameter between the homopolymer and block copolymers. Such information is critical for developing an understanding of the manner in which copolymers act as emulsifying agents and as adhesion promoters. Both subjects are of intense theoretical interest at present and the proposed studies will provide critical tests of these theories.

Along similar lines the extent to which a copolymer molecule can penetrate into a crosslinked polymer will be investigated. These studies have two goals in mind. From a fundamental viewpoint the rate at and extent to which the copolymer chains can penetrate into the crosslinked polymer will be a direct test of diffusive motions in polymers where a linear chain must reptate into the network of the crosslinked polymer. Secondly, these studies will provide information on the extent of penetration of the homopolymer chains into the crosslinked network which is critical for evaluating the effectiveness of copolymers as adhesion promoters in rubber modified and composite systems.

\section{Copolymer Mixtures}

Preliminary investigations during the past year have indicated that the surface induced ordering of mixtures of two symmetric diblock copolymers differs substantially from the behavior observed in the bulk. In the bulk it has been found that the copolymer of different molecular weights will compensate for the constraints placed on the chains by modifying the fundamental repeat period of the lamellar microphase separated morphology. The behavior of such mixtures in the vicinity of an interface appears to be quite different. Initial results indicate a preferential segregation of one of the components to the free surface and to the substrate interface. The additional perturbation placed upon the copolymer chains by the presence of an interface is sufficient to for a separation of the copolymer chains. In the 
upcoming year these studies will be extended in a more systematic manner so 25 to develop a phase diagram of the copolymer mixtures in thin films and to compare this result with current theoretical arguments.

\section{Ordering Kinetics of Copolymer at Interfaces}

It is well known that placing diblock copolymers at the interface between homopolymer will enhance the adhesion of the two polymers. However, the rate at which the adhesion is improved depends upon the amount of copolymer at the interface. It has been suggested that this is related to the rate at which the copolymer chains organize at the interface. However, no direct evidence has been found to support this argument. In keeping with the initial thrust of this proposal, in the upcoming year, the kinetics of the organization of $\mathbf{P}(\mathbf{S}-\mathrm{b}-\mathrm{MMA})$ chains at the interface between similar and dissimilar homopolymers will be studied by a combination of neutron reflectivity and dynamic secondary ion mass spectroscopy. This can be easily accomplished via selective deuteration of the copolymer chains and observing the time dependent concentration profiles. In addition, off specular neutron reflectivity will provide information on the lateral heterogeneities of the copolymer chains at the interface which will lead to an understanding of the manner in which the copolymer chains organize.

\section{The Lower Disorder to Order Transition}

In the past year studies under this proposal led to the discovery of an ordering transition which occurred for symmetric diblock copolymers upon heating. This discovery completely changes the accepted thinking on the phase behavior of diblock copolymers and warrants intense further study. In the upcoming year, studies will be geared to the investigation of this new phase behavior. Studies will focus on the quantification of the phase diagram by neutron and $x$-ray scattering and, also, by dynamic mechanical studies. It is the intent of these studies to gain an understanding of this newly found behavior and to develop set of results to guide current theoretical developments.

\section{Grazing Incidence $X$-ray Scattering}

Up to this point studies under this proposal have demonstrated that polymers which tend to order in the bulk undergo a substantial enhancement of the ordering near the surface and those molecules that are anomhous or liquid, retain the liquid-like structure. In line with this, in the upcoming year two separate lines of research will be pursued. The first deals with glassy polymers which can, under the appropriate thermal treatment, crystallize. In particular, GIXS studies will be performed on polycarbonate as a function of annealing treatment. The aim of these studies is to determine whether order is initiated from the surface. If so, what is the nature of the ordering at the surface. A second line of research that will he pursued is the effect of an external force on the ordering of polymers at surface. In particular, if the surface of the polymer is exposed to a shearing force, will this alter the structure of the polymer at the surface. These studies will focus on aromatic polyimides where a quantitative understanding has been developed in previous studies. 


\section{Publications from DOE Support Work}

1. "The Ordering of Thin Films of Symmetric Diblock Copolymers" with A. Menelle, S.F. Anastasiadis, S.K. Satija and C. F. Majkrzak, J. Colloid and Polym. Sci., Symp. Ser, 91, 97, (1993)

2. "Neutron Reflection" Encyclopedia of Materials Characterization, Manning Pub. Co., Greenwich, CT (1992), p. 660.

3. "Hairpin Configurations of Triblock Copolymers of Homopolymer Intertaces" with A.M. Mayes, B.R. Deline and T.C. Chung, Macromolecules 25, 5783 (1992).

4. "Homopolymer Distributions in Ordered Block Copolymers" with A.M. Mayes, S.K. Satija and C.F. Majkrzak, Macromolecules, $25,6523(1992)$.

5. "Distributions of Chain Ends and Junction Points in Ordered Block Copolymers" with A.M. Mayes, R.D. Johnson, S.D. Smith, S.K. Satija and C.F. Majkrzak, Macromolecules, 26, 1047 (1993)

6. "Fluctuation Effects in the ordering of Thin Diblock copolymer Films; A Reply" with A. Menelle, S.H. Anastasiadis, S.K. Satija and C.F. Majkrzak, Phys Rev Lett; 70, 1352 (1993).

7. "Very Thin Films of Symmetric Diblock Copolymers" with S.F. Anastasiadis, A. Menelle, S.K. Satija and C.F. Majkrzak, J. Colloid and Polym. Sci., 91,88 (1993) 